\title{
Chemokine receptors CXCR4 and CCR7 promote metastasis by preventing anoikis in cancer cells
}

\author{
M Kochetkova ${ }^{*, 1}$, S Kumar ${ }^{2}$ and SR McColl ${ }^{1}$
}

\begin{abstract}
Chemokine receptors are essential mediators of the metastatic spread in various cancer types; however their precise function in the development of secondary tumors remains poorly understood. We report here a novel property of the chemokine receptors CXCR4 and CCR7 in inhibiting detachment-induced cell death - anoikis, which is believed to be one of the major blocks in the metastatic spread of various neoplasms. Activation of these chemokine receptors by their respective ligands, CXCL12 and CCL21 specifically reduced the sensitivity of metastatic breast cancer cells to anoikis by a distinct mechanism of selective regulation of pro-apoptotic Bmf and anti-apoptotic Bcl-xL proteins. Consequently, functional CXCR4 and CCR7 increased cell survival in the absence of correct ECM attachment both in vitro and in vivo. We also demonstrated that preventing chemokineinduced reduction in Bmf levels significantly attenuated breast cancer metastasis in an experimental mouse model. These results provide evidence for a previously unknown axis in malignant tumors, which connects chemokine receptors with deregulated apoptosis in the absence of the appropriate cell - ECM interaction and may offer novel targets for therapeutic intervention for the treatment of metastatic breast and potentially other tumors.
\end{abstract}

Cell Death and Differentiation (2009) 16, 664-673; doi:10.1038/cdd.2008.190; published online 9 January 2009

The main cause of cancer-related deaths is the spread of tumor cells to sites distant from the primary locus and the subsequent establishment of secondary lesions. The heterogeneity and complexity of malignant transformation make it difficult to identify common molecular mechanisms governing the metastatic progression of cancer and potentially account for the lack of effective treatments for malignant cancers. Recently, members of the chemokine receptor family of G-protein-coupled receptors (GPCRs) have been assigned a major role in this process (reviewed in Zlotnik ${ }^{1}$ ). It is becoming increasingly clear that many members of this family regulate a complex milieu of interactions between tumor cells, stromal cells, tumor infiltrating leukocytes and endothelial cells and that better understanding of the specific actions of chemokines and their receptors in promoting malignancy will significantly contribute to combating cancer. ${ }^{2}$

CXCR4 and CCR7 are the major chemokine receptors found on a wide range of tumor cells and high levels of these receptors are associated with higher grade and poor prognosis of breast, lung, colon and other cancers. ${ }^{3}$ Blocking the interaction between CXCR4 and its ligand CXCL12, dramatically reduces breast and other cancer metastases in mouse models. ${ }^{4}$ Our recent results have also provided a direct correlation between functional activation of CXCR4 and CCR7 and the invasive, metastatic phenotype of breast cancer cells ${ }^{5}$ further supporting a highly important role for these molecules in tumor dissemination to distant sites. Initial studies suggested that these surface molecules induce directional migration of malignant cells along chemokine gradients towards secondary tumor sites in a manner that is similar to the process of lymphocyte homing. However, more recent evidence also supports other roles for chemokine GPCRs including cell growth, adhesion to endothelium and extravasation. $^{2}$ Yet specific, non-redundant signaling events and cellular functions directly linking these surface molecules with cancer metastasis are still largely unknown. ${ }^{6}$

Anoikis is apoptosis induced upon perturbation or loss of cell-matrix interactions. A number of recent studies have demonstrated a crucial role for anoikis in malignant transformation of various tissues, including mammary, colon, prostate, and lung epithelial tissues (reviewed in Valentijn et al. ${ }^{7}$ ). The breakdown of anoikis is believed to give cancer cells a selective advantage by increasing their survival time in the absence of appropriate extracellular matrix (ECM) attachment, that is, in the circulation or in the non-permissive environment of the distant site, thus facilitating eventual reattachment and colonization. In animal models tumors that are resistant to anoikis show a higher incidence of metastases and increased cell survival in the blood circulation. ${ }^{8}$ Recently, a direct link between cell survival and metastatic ability of viable, anoikis-resistant cells from animal blood in an orthotopic prostate cancer model has been reported. ${ }^{9}$ However, despite its potentially important role in metastasis, specific mechanisms responsible for the execution of anoikis, and its deregulation in invasive cancer cells in particular, remain poorly understood.

\footnotetext{
${ }^{1}$ School of Molecular and Biomedical Science, University of Adelaide, Adelaide, South Australia 5005, Australia and ${ }^{2}$ Centre for Cancer Biology, Hanson Institute, Frome Road, Adelaide, South Australia 5000, Australia

*Corresponding author: M Kochetkova, School of Molecular and Biomedical Science, University of Adelaide, North Terrace, Adelaide SA 5005, Australia.

Tel: + 6188303 4553; Fax: + 6188303 3337; E-mail: marina.kochetkova@adelaide.edu.au Keywords: chemokine receptors; breast cancer; metastasis; anoikis; Bmf

Abbreviations: ECM, extracellular matrix; GFP, green fluorescent protein; GPCR, G-protein coupled receptor; RFP, red fluorescent protein; shRNA, small hairpin RNA Received 15.8.08; revised 10.11.08; accepted 25.11.08; Edited by P Vandenabeele; published online 09.1.09
} 
Although chemokines and their receptors are not known to play a major role in cell death or survival signaling, there is some evidence implicating them in these processes. For example activation of chemokine receptors promotes apoptosis in neurons and $T$ cells as well as survival of glioblastoma, ovarian carcinoma, pancreatic adenocarcinoma and other cancer cells. ${ }^{10-12}$ These previous data linking some chemokine receptors with apoptosis and the correlation between functional activation of CXCR4 and CCR7 and an invasive, metastatic phenotype of breast cancer cells together with the ability of these receptors to induce cytoskeletal changes have led us to hypothesize that CXCR4 and CCR7 may influence cancer cell metastasis by regulating detachment-induced apoptosis. We describe here a novel common role for CXCR4 and CCR7 in inhibiting anoikis, specifically in metastatic breast cancer cells. We also provide evidence for a direct connection between activation of these chemokine receptors and cell death machinery and show that this axis can regulate breast cancer metastasis.

\section{Results}

CXCR4 and CCR7 ligands inhibit anoikis in metastatic breast cancer cells. In search of a functional basis of the pro-metastatic action of chemokines, we analyzed the ability of CXCR4 and CCR7 ligands to increase cell survival after a prolonged period of forced detachment in highly invasive breast epithelial tumor cells. Suspended cells were prevented from attachment or allowed to reattach in the presence or absence of CXCL12 or CCL21 following the cell death analysis. (Figure 1 and Supplementary Figure 1). All cell lines tested were sensitive to loss-of-attachment-induced apoptosis and the addition of serum, used as a control, promoted survival of both detached and attached cells (Figure 1a). However, treatment of cells with either CXCL12 or CCL21 significantly inhibited cell death only in suspended cells to a level comparable to the treatment with FCS, but had no effect on apoptosis in attached cells. Notably, both CXCL12 and CCL21 also specifically inhibited anoikis in the metastatic colon cancer cell line KM12-MS (Supplementary Figure 1). In addition, stimulation of cells with either ligand reduced detachment-induced caspase-3 activity (Figure 1b). Furthermore, we have found that chemokines interfere with the intrinsic/mitochondrial pathway of apoptosis. Treatment of suspended MDA-231 and MDA-361 cells with CXCL12 inhibited the release of cytochrome $c$ from mitochondria (Figure 1c) - a key event of the mitochondria-dependent apoptosis pathway, which mediates anoikis in most cell types. ${ }^{13}$
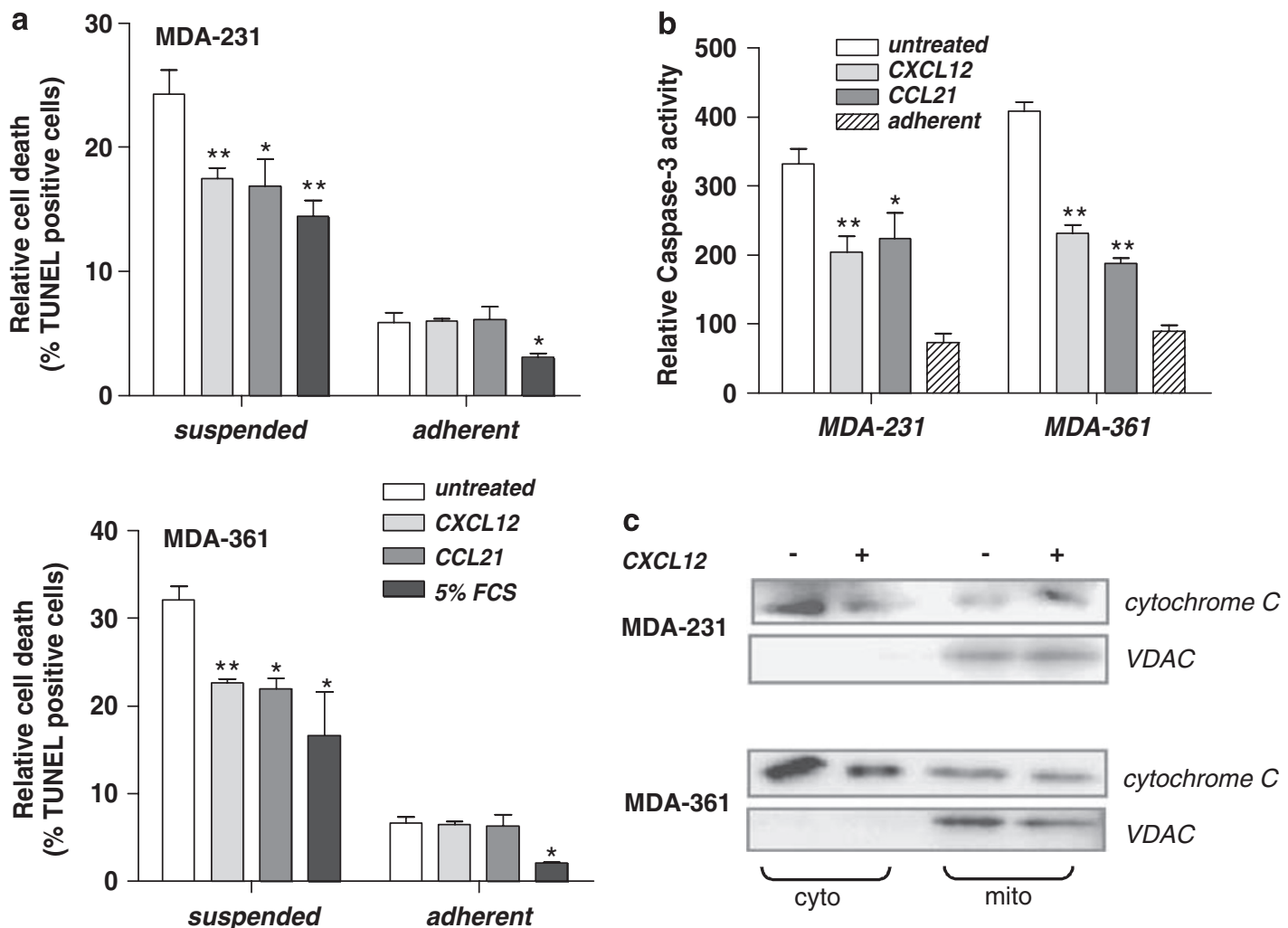

Figure 1 Ligation of CCR7 and CXCR4 inhibits detachment-induced apoptosis. (a) MDA-361 and MDA-231 cells were prevented from attaching for $48 \mathrm{~h}$, either untreated or in the presence of the CXCL12, CCL21 or 5\% FCS as indicated. Apoptotic cells were quantified by FACS using TUNEL with dUTP-TRITC. (b) Caspase 3 activity was analyzed in cell lysates after $24 \mathrm{~h}$ in suspension by colorimetric detection of $\mathrm{p}$-nitroanilide $(\mathrm{pNA})$ after cleavage from substrate DEVD-pNA. Results shown are typical of at least three independent assays in $A$ and two independent experiments in $B$, data are mean \pm S.D. For all statistical analyses in $A$ and $B{ }^{\star} P<0.05$, ${ }^{\star \star} P<0.01$ from suspended or adherent cell cultures incubated without the chemokine or FCS addition. (c) The effect of CXCL12 on the release of cytochrome c into the cytoplasm was analyzed after $24 \mathrm{~h}$ in suspension. A representative western blot from two independent experiments is shown with the mitochondria localized VDAC used as a positive control for the cellular fractionation process 
We next examined the specificity of the observed apoptosis-reducing effect. We found that ligands for both CXCR4 and CCR7 decreased anoikis only in highly invasive, metastatic breast cancer cells but failed to affect apoptosis in untransformed or non-metastatic cells with silent CXCR4 and CCR7. ${ }^{5}$ Thus detachment-induced cell death in non-invasive MDA453 (Figure 2a), MDA-134 and MCF-10A (not shown) cells was not affected by incubation with chemokines and was only reduced by the addition of serum. The selectivity of anoikisreducing function of $\mathrm{CXCL} 12$ and CCL21 was further documented by the inability of these chemokines to induce cytotoxic drug resistance. Addition of either ligand did not change the viability of MDA-231 (Figure 2b) or MDA-361 cells (not shown) treated with either etoposide or camptothecin. To further confirm that reduction in anoikis is receptor-specific we assessed the ability of chemokine ligands to inhibit cell death in detached MDA-231 cells in which either CXCR4 or CCR7 were knocked down by the retrovirally expressed shRNAs (Supplementary Figure 2). We found that the inhibition of anoikis by the chemokine ligands is highly specific for the receptor-ligand pair, as the reduction in CXCR4 or CCR7 surface levels completely abrogated the ability of their respective ligands to decrease cell death in breast cancer cells that were prevented from attachment (Figure 2c). Expression of luciferase-targeted control shRNA, which did not change the numbers of either receptor on the cell surface (Supplementary Figure 2), did not interfere with the anoikis-inhibiting function of either chemokine. Thus our
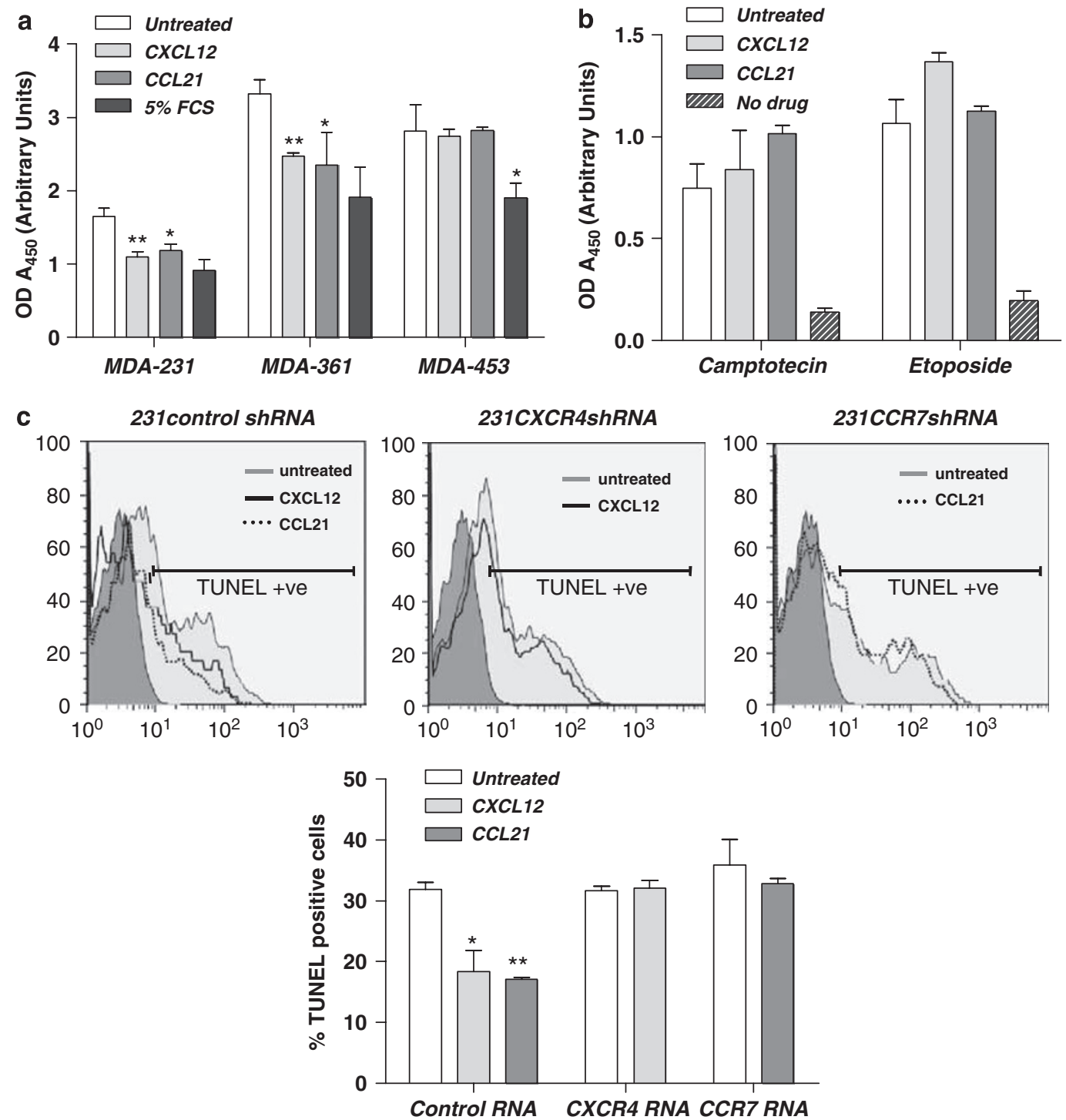

Figure 2 Anoikis-inhibiting function of CXCR4 and CCR7 is selective and receptor-specific. The effect of CXCL12 and CCL21 on DNA fragmentation as a measure of apoptosis was assessed by the cell death ELISA in suspended cells (a), or in adherent cells after the addition of cytotoxic drugs (b). (c) ShRNA-mediated knockdown of chemokine receptors abrogates inhibition of anoikis. MDA-231 cells stably expressing control luciferase, CXCR4 or CCR7-targeted shRNAs as indicated by the key, were prevented from attachment and left untreated or incubated in the presence of the CXCL12 or CCL21. Then $48 \mathrm{~h}$ later, the number of apoptotic cells was estimated by TUNEL assay. Shown are representative histograms from each cell type (top panel) and the data from at least two independent experiments (bottom panel). All data shown in a-c are representative from at least three independent experiments and are mean \pm SD with ${ }^{\star} P<0.05,{ }^{\star \star} P<0.01$ from untreated cells 
data identify a novel property of activated CXCR4 and CCR7 in reducing invasive breast (and potentially other) cancer cell death caused by loss of attachment, but not other apoptotic stimuli.

Functional CXCR4 and CCR7 increase cell survival in the absence of correct ECM contacts. We next investigated whether the ability of chemokines CXCL12 and CCL21 to inhibit anoikis will translate into an increase in survival of metastatic breast cancer cells lacking appropriate ECM attachments both in vitro and in vivo. First, MDA-231 or MDA-361 cells that were suspended for $48 \mathrm{~h}$ were allowed to reattach and grow colonies under normal culturing conditions. The number of surviving clones for all chemokine-treated cell samples was at least two times higher when compared with untreated cells, further confirming that activation of CXCR4 and CCR7 promotes cancer cell viability in the absence of attachment. (Figure 3a). With a view to further substantiate the connection between chemokine receptors, anoikis and metastasis we used shRNA-mediated knockdown (Figure 2 and Supplementary Figure 2) to investigate the ability of CXCR4 and CCR7 to influence survival of MDA-231 breast cancer cells in the incompatible ECM environment in vivo. Specific and control cell populations as indicated by the key in Figure $3 b$ were 'color-coded' with GFP or RFP, mixed in equal numbers to allow for direct comparison in every experimental animal and targeted to the lungs of SCID mice trough the lateral tail vein injection. Mice were killed at 3 and $48 \mathrm{~h}$, and the numbers of red and green fluorescent cell colonies recovered from mouse lungs after digestion and antibiotic selection were used to estimate the relative proportion of surviving cancer cells from each population. Lack of either CXCR4 or CCR7 did not significantly change the ratio of recovered specific shRNA-expressing 'red' versus control luciferase shRNAexpressing 'green' cells at $3 \mathrm{~h}$ post-injection when compared with the control mixture containing the RFP-labeled wild type suggesting that the rate of initial arrest and extravasation of all cancer cells in the lungs was relatively similar (Figure 3b) and therefore was not dependent on the activity of chemokine receptors. However, loss of either CXCR4 or CCR7 significantly reduced the number of cells recovered from mice lungs at $48 \mathrm{~h}$ post-injections with the relative proportion of specific 'red' to control 'green' colonies at an average of $1: 8$. These findings strongly implicated chemokine receptors in promoting survival of breast and potentially other cancer cells in vivo in the absence of the appropriate ECM contacts.

\section{Specific pro- and anti-apoptotic proteins mediate chemokine-induced protection from anoikis. To} understand the mechanism by which chemokines inhibit apoptosis we analyzed the expression of a panel of proapoptotic BH3-only proteins and pro-survival Bcl-2 family members in attached and detached metastatic breast cancer cells (Figure 4). Unexpectedly, with the exception of Bik, all of the tested $\mathrm{BH} 3-$ only proteins were upregulated in both cell lines tested after $24 \mathrm{~h}$ in suspension (Figure $4 \mathrm{a}$, compare a



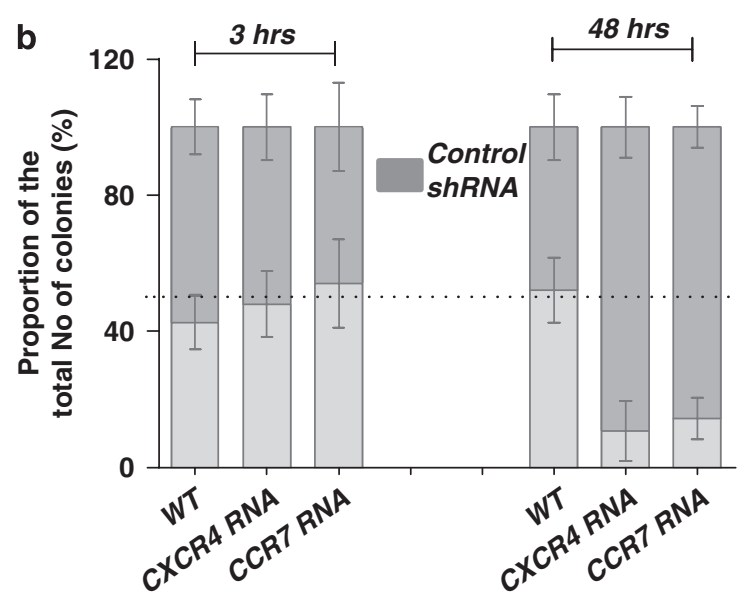

Figure 3 Chemokine receptors promote cancer cell survival in vitro and in vivo. (a) Cell survival after forced suspension was estimated by conducting a colony growth assay. Representative Giemsa-stained MDA-231 colonies (top panel) and quantification of the MDA-361 and MDA-231 colony growth after reattachment and 1 week of growth (bottom panel). Adherent cells are presented as shaded histogram. Results shown are representative of three independent experiments with ${ }^{\star} P<0.05$, ${ }^{\star \star} P<0.01$ from untreated cells. (b) GFP-labeled, luciferase shRNA-expressing control MDA-231 cells were mixed with RFP-labeled wt, CXCR4 or CCR7 shRNA-expressing cells in equal numbers as indicated and injected into the tail vein of female SCID mice. Mice were killed at indicated times post-injection; lungs were excised, digested with collagenase and resultant single cell suspensions were allowed to grow colonies in the presence of G418. Red and green fluorescent colonies were scored 2 weeks later and plotted for each experimental group $(n=3)$ as a proportion of the total number of colonies. Dotted line denotes hypothetical equal proportion. Data shown are from one of the two independent assessments with ${ }^{* *} P<0.01$ from the control, wt and luciferase shRNA-expressing cells mixture 
a

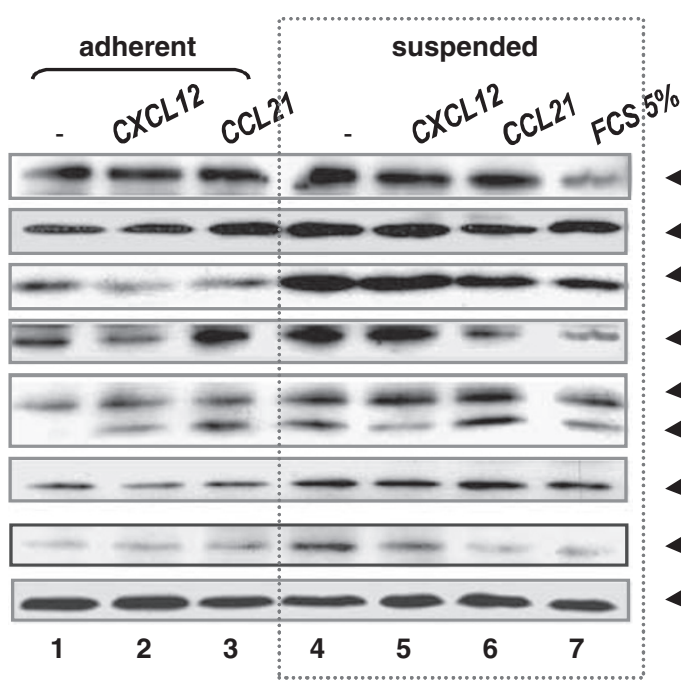

b MDA-231

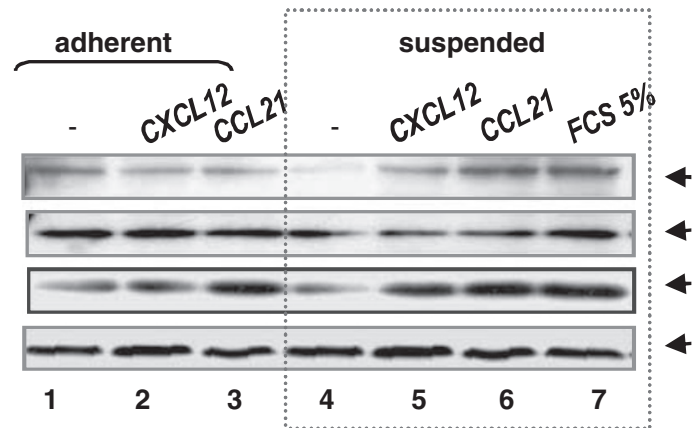

MDA-361

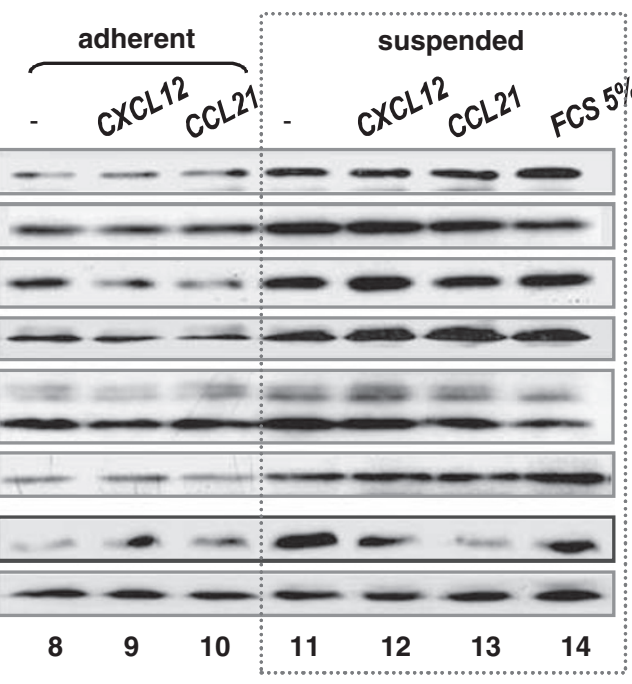

MDA-361

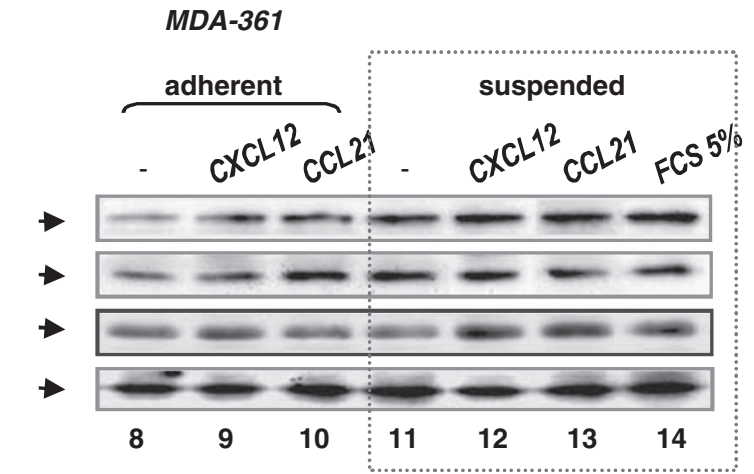

Mc/1

Bcl2

$B c l-x L$

Actin

Figure 4 Pro-apoptotic Bmf and anti-apoptotic Bcl-xL are the potential mediators of the anti-anoikis effect induced by chemokines. Breast cancer cells, adherent or suspended, were left untreated or treated as indicated for $48 \mathrm{~h}$. Expression of pro-apoptotic (a) or anti-apoptotic proteins (b) was analyzed in whole cell lysates by western blot with equal loading confirmed by equal levels of actin in the sample set. Shown are the representative western blots from at least three individual experiments for each tested protein

lanes 1 and 4, 8 and 11), emphasizing the complexity of the process of the loss-of-anchorage-induced cell death. However, only the Bmf expression was greatly and uniformly reduced by stimulation with both $\mathrm{CXCL} 12$ and CCL21 and only in suspended cells (lanes 4-6 and 11-13). Interestingly, expression of the BH3-only protein Bim, which has been suggested as a regulator of anoikis in normal as well as in cancer cells, ${ }^{13}$ showed little change after chemokine addition despite being regulated by FCS in agreement with previous reports.

Analysis of the pro-survival $\mathrm{Bcl}-2$ proteins showed that $\mathrm{Bcl}-\mathrm{xL}$, previously suggested to play an important role in anoikis, ${ }^{13}$ may also mediate chemokine-induced inhibition of anoikis in cancer cells. Although being slightly downregulated in suspended cells when compared with adherent cells, Bcl-xL expression was elevated by the addition of both CXCL12 and CCL21 to detached MDA-231 and MDA-361 cells (Figure 4b). Interestingly, the levels of $\mathrm{Bcl}-2$, previously implicated in anoikis process in numerous cell types, ${ }^{13}$ did not change under any of the conditions tested.

These results provide evidence for a specific and novel link between activation of chemokine receptors and regulation of the $\mathrm{Bcl}-2$ protein family members in metastatic breast cancer cells after the loss of the ECM attachment.

JNK, PI3K and NF- $\kappa$ B-signaling pathways mediate inhibition of anoikis by chemokine ligands. To identify common downstream effectors that participate in anoikis inhibition by chemokines we used pharmacological inhibitors and dominant-negative mutants to block specific signaling pathways. We therefore chose to investigate a role of specific signaling mediators activated downstream of both CCR7 and CXCR4 in cancer cells that have been also implicated in the regulation of anoikis as well as in metastatic spread (Figure 5). To this end we assessed the contribution of selected signaling pathways to the capacity of ligated CXCR4 and CCR7 to modulate specific Bcl-2 family members and to inhibit anoikis in MDA-361 and MDA-231 cells. Pretreatment of cells with pertussis toxin (PTX), an inhibitor of GPCR/Gi coupling, reversed chemokine-induced protection from apoptosis (Figure $5 \mathrm{a}$ ) as well as modulation of the expression of $\mathrm{Bcl}-2$ proteins (Figure $5 \mathrm{~b}$ ) confirming the involvement of $G_{i}$ subunit. Addition of the $P I 3$ kinase inhibitor LY 294002 and a c-Jun N-terminal kinase (JNK) inhibitor 

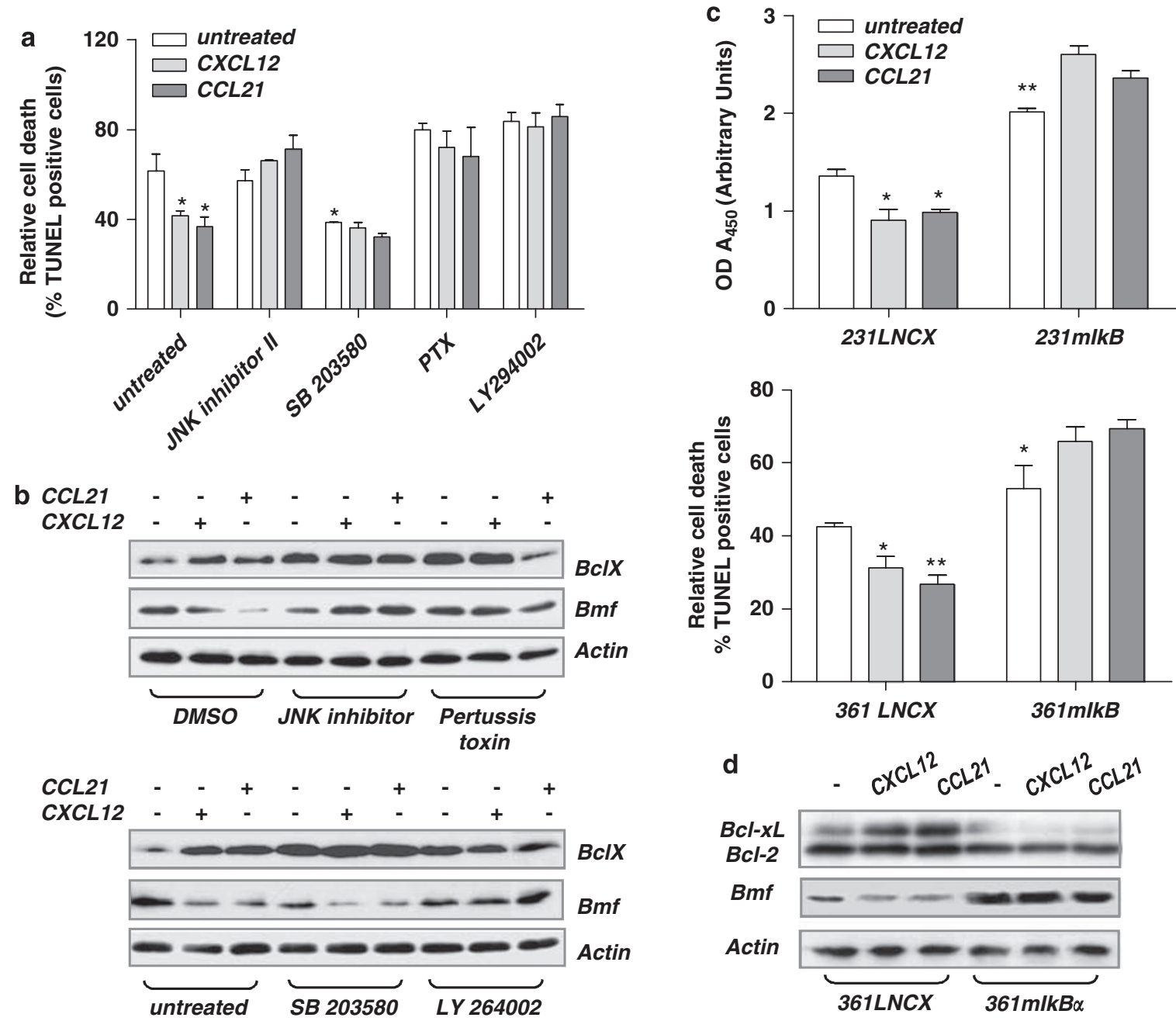

Figure 5 Multiple-signaling pathways mediate anoikis inhibition by CXCR4 and CCR7. (a and $\mathbf{b}$ ) Cells were pre-incubated with the specified inhibitors and then suspended in media only or in the presence of CXCL12 or CCL21. (a) The extent of anoikis in MDA-361 cells after $48 \mathrm{~h}$ in suspension was estimated by a TUNEL assay with the data presented as mean \pm S.D., ${ }^{*} P<0.05$ from cells incubated in media alone. (b) MDA-361 whole cell lysates were subjected to western blot analysis with Bmf or Bcl-xL antibodies. Actin was assessed as a loading control. (c) The extent of anoikis in mutant IkB-a (mlkB)- or vector control (LNCX)-transduced cells was determined by TUNEL (MDA-231 cells, upper panel) or DNA fragmentation ELISA (MDA-231 cells, lower panel) assays. Data presented are mean \pm S.D. with ${ }^{*} P<0.05,{ }^{* \star} P<0.01$ from vector control-expressing cells incubated in media alone. (d) Whole cell lysates from detached MDA-361 cells, expressing mutant lkB or vector control were subjected to western blot analysis with antibodies to the indicated proteins with actin expression used as a loading control. Results, shown in a-d each are representative from at least three independent experiments

similarly blocked chemokine-dependent reduction in anoikis and the underlying $\mathrm{Bcl}-\mathrm{xL}$ and $\mathrm{Bmf}$ regulation without significantly affecting overall levels of cell death. In contrast, inhibition of p38 MAPK pathway did not appreciably alter the inhibition of cell death by chemokines but significantly reduced the basal levels of anoikis in MDA361 (Figure 5a) and MDA-231 (not shown) cells. This effect most likely resulted from the changes in Bcl-2 proteins as pretreatment of suspended cells with SB 203580 led to an increase in $\mathrm{Bcl}-\mathrm{xL}$ levels concomitantly with a reduction in Bmf (Figure 5b).

In an attempt to more precisely delineate the role of JNK in the chemokine-induced inhibition of anoikis we assayed the effect of CXCL12 or CCL21 on the cleavage of pro-apoptotic Bid and the production of its active truncated form tBid - the process that is mediated by JNK activation. ${ }^{14}$ Western blot analysis with polyclonal antibodies that recognize tBid failed to detect any significant changes in Bid degradation after addition of chemokines to suspended MDA-231 or MDA-361 cells (not shown), suggesting an involvement of a different and potentially novel signaling pathway downstream of JNK.

The facts that NF- $\kappa \mathrm{B}$ activation inhibits anoikis in epithelial cells ${ }^{15}$ and that activated CXCR4 and CCR7 among other G-protein-coupled receptors induce NF- $\kappa$ B activation in leukocytes and other cells, ${ }^{16}$ prompted us to investigate the involvement of NF- $\kappa \mathrm{B}$ in the inhibition of apoptosis by CXCL12 and CCL21. First, we found that the addition of both ligands led to an increase in NF- $\kappa \mathrm{B}$-driven luciferase reporter gene activity and phosphorylation of $\mathrm{I}_{\kappa} \mathrm{B}-\alpha$ over basal levels (Supplementary Figure $3 A$ and $B$ ). Notably, in contrast with 
previous reports investigating anoikis in untransformed epithelial cells, ${ }^{15}$ we did not detect any raise in NF- $\kappa$ B activity after prolonged suspension most likely due to a high constitutive activation of NF- $\kappa \mathrm{B}$ in invasive cancer cells used in this study. ${ }^{17}$ However, the effect of chemokines on cell survival (Figure $5 \mathrm{c}$ ) as well as $\mathrm{Bcl}-\mathrm{xL}$ and $\mathrm{Bmf}$ modulation was reversed in both MDA-361 (Figure 5d) and MDA-231 (not shown) after blocking activation of NF- $\kappa$ B by the expression of a mutant 'super-repressor' of $I_{\kappa} \mathrm{B}-\alpha$ (Supplementary Figure $3 C)$, linking this transcriptional pathway to anoikis regulation by CXCR4 and CCR7. Inhibition of NF- $\kappa$ B also lead to a significant increase in overall cell death in both cell lines irrespective of chemokine treatment, which probably resulted from concomitant increase in $\mathrm{Bmf}$ and reduction in $\mathrm{Bcl}-\mathrm{xL}$ levels (Figure $5 \mathrm{~d}$ ) but not Bcl-2 levels which were only slightly affected by the NF- $\kappa$ B inhibition.

Upregulation of Bmf prevents the inhibition of anoikis by chemokines. To investigate a causal relationship between Bmf expression and its inhibition by chemokine receptors in the loss-of-attachment-induced apoptosis, we forced MDA231 and MDA-361 to stably express this BH3-only protein through retroviral transduction. Contrary to the earlier reports $^{18} \mathrm{Bmf}$ overexpression did not result in any significant increase in overall cell death when compared with the vector only controls in either suspended (Figure 6a) or attached cells (not shown). This discrepancy could be explained by differences in the apoptosis susceptibility of the normal or untransformed cells used previously, and the more apoptosis-resistant metastatic cancer cells, used in this study. Elevated levels of Bmf also did not significantly change the cellular proliferation rate (Supplementary Figure 4). However, preventing the chemokine-induced downregulation of Bmf by its stable expression completely abrogated the ability of CXCL12 or CCL21 to inhibit anoikis in both cell lines, confirming the functional importance of the newly delineated axis between chemokine receptors and this BH3-only pro-apoptotic protein.

To investigate the role of $\mathrm{Bmf}$ in a metastatic process more directly, we next used a dual fluorescence assay in an SCID
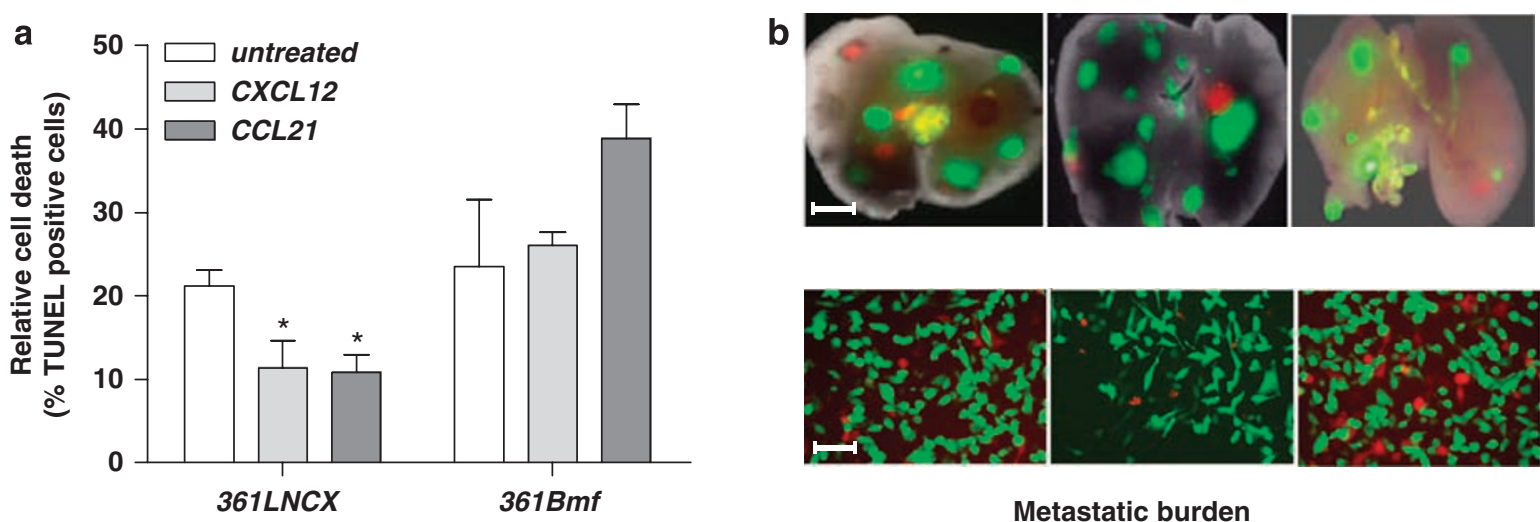

Metastatic burden
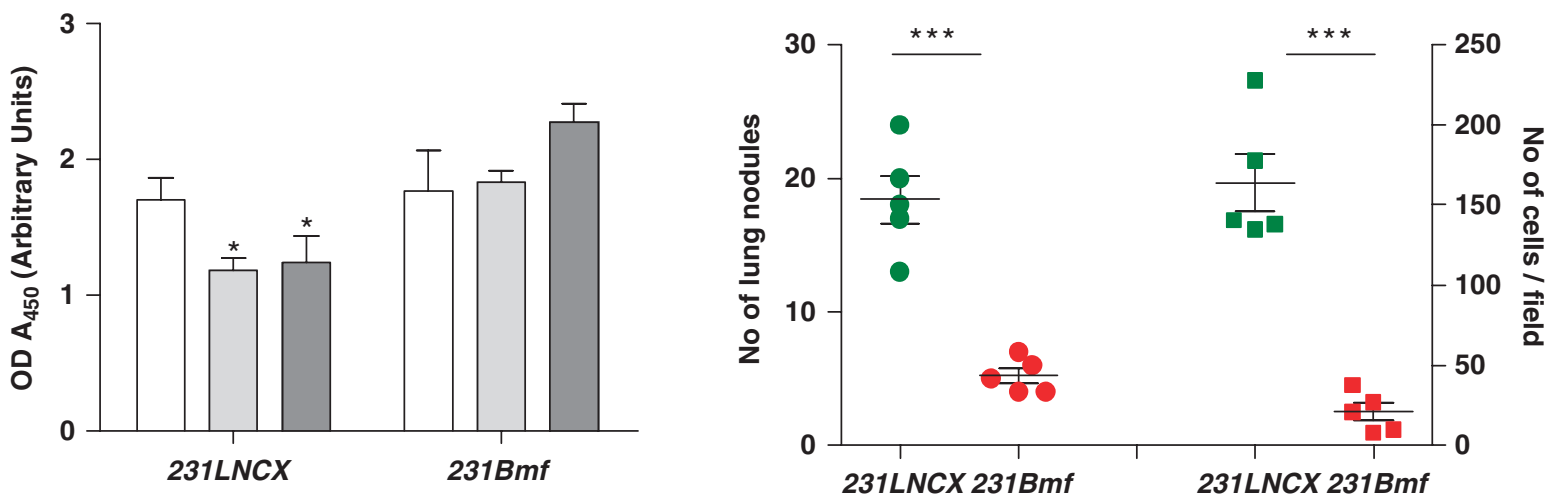

Figure 6 Increased Bmf expression abrogates chemokine-induced inhibition of anoikis in vitro and experimental metastasis of breast cancer in vivo. (a) The effect of Bmf expression on detachment-induced apoptosis was estimated by FACS-based TUNEL assay in MDA-361 cells (upper graph) and DNA fragmentation analysis in MDA-231 cells (lower graph). Cells expressing vector control (LNCX) or Bmf (Bmf) were maintained in suspension for $48 \mathrm{~h}$ and subjected to the indicated apoptosis assay. Data shown are mean \pm S.D. and are representative of at least three independent experiments with ${ }^{*} P<0.05$ from the cells left untreated. (b) The effect of Bmf expression on experimental lung metastasis: upper panel, a 1:1 mixture of the GFP-labeled vector control-expressing 231LNCX and the RFP-labeled Bmf-expressing 231Bmf cells was injected into the tail veins of female SCID mice. Whole lungs were excised and metastatic lesions were visualized by fluorescent microscopy. Shown are three out of five representative overlay images of excised lungs, which were recorded under bright field and fluorescent filters. Scale bar, $1.8 \mathrm{~mm}$. Middle panel, excised lungs were digested with collagenase, and human cancer cells were allowed to outgrow in the presence of an antibiotic. Shown are overlay images of the representative fields from three out of five randomly selected digested lungs, recorded under red and green fluorescent filters. Scale bar, $100 \mu \mathrm{m}$. Bottom panel, Quantification of the GFP- versus RFP-positive lung metastatic colonies $(\boldsymbol{Q}$, left $y$ axis) and human breast cancer cells $(\boldsymbol{\square}$, right $y$ axis) recovered from digested lungs. All data are representative from one of two independent experiments and shown are mean \pm S.D. from five mice, ${ }^{* * \star} P<0.01$ 
mouse model of experimental breast cancer metastasis to the lung. GFP-labeled vector control and the RFP-tagged Bmfexpressing MDA-231 cells were mixed in equal numbers and injected into the tail vein of the female SCID mice. Evaluation of metastases in mouse lungs after 10 weeks of growth showed an overwhelming dominance of green fluorescent lesions from control cells with almost no visible red fluorescent colonies, which were indicative of the Bmf-transduced cells (Figure 6b). In addition, we have digested excised lungs with collagenase and purified metastasized breast cancer cells by antibiotic selection. In agreement with the results of the lung surface colony assay, the total lung metastatic burden was heavily skewed toward GFP-tagged control cells with an average five-fold reduction in a number of the Bmf-expressing RFP-positive cells (Figure 6b). These findings show that a modest increase in anoikis in vitro through prevention of chemokine-induced downregulation of the pro-apoptotic Bmf (Figure 6a) can translate into a dramatic reduction in the metastatic capacity of breast cancer cells in vivo.

\section{Discussion}

Metastatic spread is believed to be a very inefficient process where, of the presumed millions of cells that are shed into circulation by a primary tumor very few will establish secondary lesions. ${ }^{19,20}$ This is likely due to the fact that only a small proportion of cells that reach secondary sites will survive beyond the first $24 \mathrm{~h} .{ }^{21}$ The vast majority of these cells will undergo apoptosis with the loss of attachment probably being the main apoptotic stimulus and therefore factors that promote survival of the breakaway tumor cells can effectively be considered as critical determinants of the cancer metastatic process. A multitude of studies have clearly implicated chemokine receptors CXCR4 and CCR7 as metastasispromoting molecules. We describe here a previously unknown function for these chemokine receptors in inhibiting detachment-induced cell death specifically in highly invasive cancer cells. Thus systemic chemokine ligands may influence the persistence of detached tumor cells in the circulation or immediately after extravasation, which is considered to be one of the most critical steps in a metastatic cascade. This novel role may help to explain the critical importance of chemokine receptors in metastatic dissemination of vastly diverse cancer types. These findings may also have important implications for devising novel therapies for metastatic tumors. The fact that an anoikis-inhibiting function of both CXCR4 and CCR7 was found to be uniform in a number of metastatic cancer cell lines potentially points at an important novel common molecular mechanism that is involved in the malignant spread of tumor cells to secondary sites.

We found that chemokines CCL21 and CXCL12 exerted a selective effect on cell survival by reducing apoptosis caused only by loss of attachment, and not by serum deprivation or the addition of cytotoxic drugs. By using a mouse metastatic model we were also able to establish that CXCR4 or CCR7 activity is required by breast cancer cells to survive in vivo within the lungs. Interestingly, adhesion to endothelium and extravasation of cancer cells were the two major steps in a metastatic cascade that were previously suggested to be affected by chemokine receptor activation ${ }^{22}$ with the evidence mainly derived from in vitro cellular assays. In contrast with these reports our in vivo experiments show that the silencing of either CXCR4 or CCR7 did not change the rate of the initial arrest of cancer cells but critically reduced the number of metastatic breast cancer cells persisting in the lung 2 days after intravenous injection.

We propose therefore that ligation of CXCR4 or CCR7 can specifically compensate for the absence of cellular survival signals after the loss of an ECM attachment or in a context of inappropriate matrix environment at distant organs and thus facilitate metastatic dissemination. Recent reports demonstrating that ligands for CCR7 are expressed in human lungs and brain ${ }^{23,24}$ - the two common secondary sites besides the lymph nodes that are targeted by the breast cancer - together with the fact that CXCR4 ligand CCL12 is also produced by these and other organs, provide further support for this hypothesis.

Integrin attachment to the ECM provides cell survival signals through direct and indirect connections to the actin cytoskeleton, growth factor receptors, and intracellular signal transduction cascades. ${ }^{25}$ Cytoskeletal changes induced by various stimuli have also been shown to regulate integrin activity and the sensitivity to anoikis. ${ }^{26}$ In turn chemokine receptors are tightly linked to cytoskeleton remodeling and integrin receptor activation in migrating leukocytes and induce actin polymerization in various cancer cell types. ${ }^{27,28}$ Therefore, it is plausible to suggest that chemokine stimulation of metastatic tumor cells, either detached or surrounded by incompatible ECM, leads to actin restructuring, which in combination with other survival signals inhibit anoikis. The fact that CXCR4 is capable of directly interacting with myosin $I \mathrm{~A},{ }^{29}$ which has been implicated in the formation of focal adhesions in HeLa cells further supports this hypothesis. Survival cues from trans-activated growth factor receptors (e.g., see Porcile et al. ${ }^{30}$ ) may also contribute to the anoikisinhibiting property of chemokines.

In addition, we have delineated a novel molecular link between activation of chemokine receptors and regulation of the $\mathrm{Bcl}-2$ protein family members in metastatic breast cancer cells after the loss of the ECM attachment. Our results strongly suggest that a decrease in Bmf and an increase in $\mathrm{Bcl}-\mathrm{xL}$ levels may be the main contributors to the chemokinemediated inhibition of anoikis. This is in accordance with previous reports implicating these $\mathrm{Bcl}-2$ proteins in detachment-induced apoptosis. ${ }^{13,18,31}$ Simultaneous changes in these two proteins also suggest a non-linear signaling cascade leading to the inhibition of anoikis by active chemokine receptors in cancer cells. In additional, alteration of the phosphorylation state of some $\mathrm{BH}$-only proteins including Bim, an important anoikis modulator, ${ }^{13}$ was also proposed as a mechanism to control their pro-apoptotic function. Although we have demonstrated that the only proapoptotic $\mathrm{BH} 3-o n l y$ protein showing alterations in expression was Bmf protein, the regulation of the pro-apoptotic activity of other BH3-only proteins by chemokines through the changes in phosphorylation cannot be ruled out at this stage. It has to be noted however, that we have not detected any changes in phosphorylation of BAD at $\operatorname{Ser}^{136}$ after chemokine stimulation (not shown) which was described previously for BAD and CXCL12 $2^{32}$ in lymphocytes. 
A number of signaling pathways have been implicated in anoikis regulation. We have found that the changes in $\mathrm{Bcl}-2$ family proteins and accompanying inhibition of the loss-ofattachment-induced apoptosis by chemokine ligands is mediated by multiple-signaling pathways, with the somewhat predictable participation of $\mathrm{PI} 3 \mathrm{~K}$ and the less expected involvement of JNK (Figure 3). Interestingly, our findings add to a small but actively growing body of evidence in support of a non-canonical apoptosis-inhibiting function of JNK (for e.g., see Kook et al. and Kuntzen et al. ${ }^{33,34}$ ) providing further evidence for the recently emerged paradigm of dual proapoptotic or pro-survival activity of this SAP kinase which depends on the cell type and/or system studied. We have also found that blocking p38 activation leads to significant changes in basal levels of the detachment-induced apoptosis in parallel with the inhibition of Bmf upregulation and $\mathrm{Bcl}-\mathrm{xL}$ downregulation, similar to that recently reported for TGF $\beta$-induced cell death. ${ }^{35}$ Based on these observations and bearing in mind an important function recently assigned to p38 MAPK in anoikis $^{36}$ it is intriguing to hypothesize that activation of the chemokine receptors in cells within an unfavorable ECM context may lead to an impairment of p38 activity and a decrease in cell death.

We have also described previously unknown role for the pro-apoptotic Bmf protein in regulating inhibition of anoikis by chemokine receptors. Our results show that in highly transformed epithelial cells, Bmf is a necessary but not sufficient cell death-inducing factor. Thus, increasing the levels of this protein did not lead to any significant changes in cell death in vitro, however preventing its downregulation resulted in a dramatic decrease in experimental metastatic lesions in vivo potentially suggesting that a number of proapoptotic signals might need to be involved simultaneously to effectively kill metastatic tumor cells. Notably, overexpression of Bmf did not affect the ability of chemokines to increase Bcl-xL levels (Supplementary Figure 5), indicating that ultimately it is likely the ratio of these two proteins, controlled by chemokine receptors in suspended cells, that determines the balance between cell death and cell survival, an observation that is in agreement with the current understanding of the apoptotic regulatory mechanisms. ${ }^{37}$ Therefore, decreasing Bmf levels under conditions of non-complementary ECM or lost anchorage may be an important survival-stimulating signal that is generated from ligated CXCR4 and CCR7. This may represent a novel molecular mechanism underlying the metastasis-promoting function of these receptors. Importantly, our results also reveal a previously unknown role for the pro-apoptotic Bmf protein in suppressing breast cancer metastasis thus taking further recent observations, which implicated this protein as a mediator of mammary gland involution and a tumor suppressor in mammary epithelial ${ }^{18}$ and lymphoid ${ }^{38}$ cells.

In conclusion, a novel function for chemokine receptors CXCR4 and CCR7 in inhibiting anoikis will help to explain the immense importance that has been implied for these molecules in almost every type of malignant carcinoma and other metastatic cancers. These findings may have wide implications for the understanding of the complex metastatic process in breast and potentially other cancers and also call for caution in the application of the chemokine-based anti-cancer vaccines (for e.g., see Okada et al. ${ }^{39}$ ), which may inadvertently lead to an increase in metastatic dissemination.

\section{Materials and Methods}

Cell lines, antibodies and reagents. The human cancer cell lines, MCF$10 \mathrm{~A}$ and the human embryonic kidney cell line HEK 293T were all obtained from the ATCC. Cells were grown at $37^{\circ} \mathrm{C}$ in $5 \% \mathrm{CO}_{2}$ in a humidified atmosphere according to the supplier's instructions. To analyze the effect of a forced detachment, for all assays freshly trypsinized cells were seeded in DMEM culture media without FCS onto poly-HEMA-coated dishes from Corning Inc., at a cell density of $2.5 \times 10^{5}$ cells per ml. Mcl-1, Bid, and Bim rabbit monoclonal antibodies were from Epitomics; $\mathrm{Bcl}-\mathrm{x}, \mathrm{Bcl}-2$, Bad and Nip-1 mouse monoclonal antibodies were from BD Transduction laboratories; Bik rabbit polyclonal antibodies were from Cell Signaling Technologies. Bmf rat monoclonal antibodies $12 \mathrm{E} 10$ and $9 \mathrm{G} 10$ were a kind gift from Professor A Strasser (WEHI, Melbourne, Australia). PTX used at $10 \mathrm{ng} / \mathrm{ml}$ was from Sapphire Bioscience, NSW, Australia. SB 203580 was used at $5 \mu \mathrm{M}$, JNK inhibitor at $10 \mu \mathrm{M}$ and LY294002 at $30 \mu \mathrm{M}$ (all from Calbiochem). Synthetic chemokine ligands were from the Biomedical Research Centre, University of British Columbia; Vancouver, Canada. CXCL12 was used at $100 \mathrm{ng} / \mathrm{ml}$ and $\mathrm{CCL} 21$ at $200 \mathrm{ng} / \mathrm{ml}$ final concentrations.

MDA-231 cells with the reduced CCR7 surface levels were generated through infection of cells with retroviral particles with integrated shRNA that targeted nucleotides 270-289 from the coding region of CCR7 mRNA effectively as was previously described for the CXCR4 knockdown in the same cells. MDA-231 cells, expressing shRNA-targeting Renilla luciferase were used as a negative control. ${ }^{40}$ Three individual clones, in which CXCR4 or CCR7 surface levels were reduced by at least $65 \%$, were pooled for further assays (Supplementary Figure 2A). Control luciferase shRNA-expressing cells were used as a polyclonal population.

Western blot. Western blot analysis was performed as described earlier. ${ }^{5}$ For the cytochrome $c$ release assay cells were suspended for $24 \mathrm{~h}$ and the cytoplasmic and mitochondrial protein fractions were separated using Mitochondria Isolation kit (Pierce).

Apoptosis assays. Apoptotic cells were quantified using the cell death detection ELISA kit and terminal deoxynucleotidyl transferase-mediated dUTPTRITC nick-end labeling assay (TUNEL Assay) kit (both from Roche) according to the manufacturer's instructions. Caspase-3 activity was determined using a Colorimetric Assay Kit (BioVision).

In vitro survival assay. After $48 \mathrm{~h}$ of culture on poly-HEMA-coated dishes $100 \mu$ aliquots of cell suspensions were plated in triplicate on uncoated 6-well tissue culture plates and grown for 1 week in complete growth medium. Cells were fixed in $3.7 \%$ formaldehyde, stained with Giemsa and colonies $>50 \mu \mathrm{m}$ in diameter were scored as positive.

Retroviral transduction. GFP and RFP were subcloned into Xhol and Notl sites of the retroviral expression vector pLNCX2 from Clontech. Construction of the pLNCX2-based retroviral vectors encoding full length Bmf and mutant 'superrepressor' $\left.\right|_{\kappa} \mathrm{B}-\alpha$ is described in SI Experimental Procedures. VSV-G-pseudotyped retroviruses were generated and breast cancer cell lines were infected as described in Akekawatchai et $a l .{ }^{40}$ Polyclonal populations were selected in the presence of G418 for 1 week.

In vivo survival and metastasis assays. Six to eight-week female $\mathrm{CB}-17$ SCID mice were purchased from the Animal Resource Centre (Perth, WA, Australia). SCID mice were housed in pathogen-free conditions in the immunocompromised barrier rodent facility at the Adelaide University Animal House (Adelaide, SA, Australia). All procedures performed on mice were in accordance with the strict guidelines of the NH\&MRC.

For the survival assay GFP-labeled control, luciferase shRNA-expressing MDA231 cells were mixed with RFP-labeled wt, CXCR4 or CCR7 shRNA-expressing cells in equal numbers. 'Color-coded' cell mixtures of $6 \times 10^{5}$ total cells suspended in $200 \mu \mathrm{l}$ of PBS were injected into the tail vein of three mice for each experimental group per time point. Mice were killed by $\mathrm{CO}_{2}$ inhalation at 3 and $48 \mathrm{~h}$ post-injection, lungs were perfused with PBS, excised, finely minced and incubated for $1 \mathrm{~h}$ in digestion media (RPMI with $150 \mathrm{U} / \mathrm{ml}$ collagenase type IV, and $33 \mathrm{U} / \mathrm{ml}$ of bovine 
pancreas grade II DNasel). Single cell suspensions were prepared by repeatedly aspirating the mixture through a $20 \mathrm{ml}$ syringe before filtering through a nylon mesh. Cells were then pelleted at 300 r.p.m., washed in PBS, and plated on $6 \mathrm{~cm}$ tissue culture plates in complete media with $500 \mathrm{ng} / \mathrm{ml}$ of $\mathrm{G} 418$ to select for human cancer cells. Recovered human cancer cells were allowed to outgrow and form colonies for a further 2 weeks. Red and green fluorescent colonies were then scored using a Leica stereo fluorescence dissection microscope model MZ16FA.

For the metastasis assay five SCID mice each received tail vein injections of a total $5 \times 10^{5}$ of $1: 1$ cell mixtures of the GFP-labeled vector control and the RFPlabeled Bmf-expressing MDA-231 cells, suspended in $200 \mu$ l of PBS. Mice were killed by $\mathrm{CO}_{2}$ inhalation at week 10 . Lungs were perfused with PBS, harvested and imaged by light and fluorescence microscopy using a Leica stereo fluorescence dissection microscope model MZ16FA. Images were processed by Metamorph Version 3.0 software (Universal Imaging Corp., Downingtown, PA, USA). The right lung was minced and digested with collagenase as described above. Single cell suspensions were then placed in $10 \mathrm{~cm}$ tissue culture plates in complete media with $500 \mathrm{ng} / \mathrm{ml}$ of G418 to select for human cancer cells. After 1 week of selection images of five independent fields were recorded and red and green fluorescent cells were counted to quantify lung metastatic burden.

Statistical analysis. Statistical analysis was performed on GraphPad Prism version 5.0 for windows XP (GraphPad Software, San Diego, CA, USA).

Acknowledgements. We thank Dr. Jane Holland, presently at MaxDelbrueck-Center for Molecular Medicine, Berlin, for invaluable help with animal experiments and Gemma Sharp, presently at the University of Cambridge for assistance with the experiments on NF- $\kappa \mathrm{B}$. The work was supported by grants from the National Health and Medical Research Council of Australia.

1. Zlotnik A. Involvement of chemokine receptors in organ-specific metastasis. Contrib Microbiol 2006; 13: 191-199.

2. Kakinuma T, Hwang ST. Chemokines, chemokine receptors, and cancer metastasis. J Leukoc Biol 2006; 79: 639-651.

3. Zlotnik A. Chemokines and cancer. Int J Cancer 2006; 119: 2026-2029.

4. Ruffini PA, Morandi P, Cabioglu N, Altundag K, Cristofanilli M. Manipulating the chemokine-chemokine receptor network to treat cancer. Cancer 2007; 109: 2392-2404.

5. Holland JD, Kochetkova M, Akekawatchai C, Dottore M, Lopez A, McColl SR. Differential functional activation of chemokine receptor CXCR4 is mediated by $\mathrm{G}$ proteins in breast cancer cells. Cancer Res 2006; 66: 4117-4124.

6. Zlotnik A. New insights on the role of CXCR4 in cancer metastasis. J Pathol 2008; 215: 211-213.

7. Valentijn AJ, Zouq N, Gilmore AP. Anoikis. Biochem Soc Trans 2004; 32 (Part3): 421-425

8. Wewer UM, Shaw LM, Albrechtsen R, Mercurio AM. The integrin alpha 6 beta 1 promotes the survival of metastatic human breast carcinoma cells in mice. Am J Pathol 1997; 151: 1191-1198.

9. Glinskii $A B$, Smith $B A$, Jiang $P$, Li XM, Yang M, Hoffman RM et al. Viable circulating metastatic cells produced in orthotopic but not ectopic prostate cancer models. Cancer Res 2003; 63: 4239-4243.

10. Rollins BJ. Inflammatory chemokines in cancer growth and progression. Eur J Cancer 2006; 42: 760-767.

11. Kaul M, Lipton SA. Signaling pathways to neuronal damage and apoptosis in human immunodeficiency virus type 1-associated dementia: Chemokine receptors, excitotoxicity, and beyond. J Neurovirol 2004; 10 (Suppl 1): 97-101.

12. Ajuebor MN, Aspinall Al, Zhou F, Le T, Yang Y, Urbanski SJ et al. Lack of chemokine receptor CCR5 promotes murine fulminant liver failure by preventing the apoptosis of activated CD1d-restricted NKT cells. J Immunol 2005; 174: 8027-8037.

13. Martin SS, Vuori K. Regulation of Bcl-2 proteins during anoikis and amorphosis. Biochim Biophys Acta 2004; 1692: 145-157.

14. Lauricella M, Emanuele S, D'Anneo A, Calvaruso G, Vassallo B, Carlisi D et al. JNK and AP-1 mediate apoptosis induced by bortezomib in HepG2 cells via FasL/caspase-8 and mitochondria-dependent pathways. Apoptosis 2006; 11: 607-625.

15. Yan SR, Joseph RR, Rosen K, Reginato MJ, Jackson A, Allaire N et al. Activation of NFkappaB following detachment delays apoptosis in intestinal epithelial cells. Oncogene 2005; 24: 6482-6491.

16. Ye RD. Regulation of nuclear factor kappaB activation by G-protein-coupled receptors $J$ Leukoc Biol 2001; 70: 839-848.
17. Nakshatri $H$, Bhat-Nakshatri $P$, Martin DA, Goulet Jr RJ, Sledge Jr GW. Constitutive activation of NF-kappaB during progression of breast cancer to hormone-independent growth. Mol Cell Biol 1997; 17: 3629-3639.

18. Schmelzle T, Mailleux AA, Overholtzer M, Carroll JS, Solimini NL, Lightcap ES et al. Functional role and oncogene-regulated expression of the BH3-only factor Bmf in mammary epithelial anoikis and morphogenesis. Proc Natl Acad Sci USA 2007; 104: 3787-3792.

19. Chambers AF, Groom AC, MacDonald IC. Dissemination and growth of cancer cells in metastatic sites. Nat Rev Cancer 2002; 2: 563-572.

20. Wong CW, Lee A, Shientag L, Yu J, Dong Y, Kao G et al. Apoptosis: an early event in metastatic inefficiency. Cancer Res 2001; 61: 333-338.

21. Hedley BD, Vaidya KS, Phadke P, Mackenzie L, Dales DW, Postenka CO et al. BRMS1 suppresses breast cancer metastasis in multiple experimental models of metastasis by reducing solitary cell survival and inhibiting growth initiation. Clin Exp Metastasis 2008; 25: 727-740.

22. Ben-Baruch A. Organ selectivity in metastasis: regulation by chemokines and their receptors. Clin Exp Metastasis 2008; 25: 345-356.

23. Pierce EM, Carpenter K, Jakubzick C, Kunkel SL, Evanoff H, Flaherty KR et al. Idiopathic pulmonary fibrosis fibroblasts migrate and proliferate to CC chemokine ligand 21. Eur Respir J 2007; 29: 1082-1093.

24. Krumbholz M, Theil D, Steinmeyer F, Cepok S, Hemmer B, Hofbauer M et al. CCL19 is constitutively expressed in the CNS, up-regulated in neuroinflammation, active and also inactive multiple sclerosis lesions. J Neuroimmunol 2007; 190: 72-79.

25. Hehlgans S, Haase M, Cordes N. Signalling via integrins: implications for cell survival and anticancer strategies. Biochim Biophys Acta 2007; 1775: 163-180.

26. Deschesnes RG, Patenaude A, Rousseau JL, Fortin JS, Ricard C, Cote MF et al. Microtubule-destabilizing agents induce focal adhesion structure disorganization and anoikis in cancer cells. J Pharmacol Exp Ther 2007; 320: 853-864

27. Maqueda A, Moyano JV, Gutierrez-Lopez MD, Ovalle S, Rodriguez-Frade JM, Cabanas C et al. Activation pathways of alpha4beta1 integrin leading to distinct T-cell cytoskeleton reorganization, Rac1 regulation and Pyk2 phosphorylation. J Cell Physiol 2006; 207 : 746-756.

28. Cohen-Hillel E, Yron I, Meshel T, Soria G, Attal H, Ben-Baruch A. CXCL8-induced FAK phosphorylation via CXCR1 and CXCR2: cytoskeleton- and integrin-related mechanisms converge with FAK regulatory pathways in a receptor-specific manner. Cytokine 2006; 33 : $1-16$

29. Rey M, Vicente-Manzanares M, Viedma F, Yanez-Mo M, Urzainqui A, Barreiro $O$ et al. Cutting edge: association of the motor protein nonmuscle myosin heavy chain-IIA with the C terminus of the chemokine receptor CXCR4 in T lymphocytes. J Immunol 2002; 169: 5410-5414.

30. Porcile C, Bajetto A, Barbieri F, Barbero S, Bonavia R, Biglieri M et al. Stromal cell-derived factor-1alpha (SDF-1alpha/CXCL12) stimulates ovarian cancer cell growth through the EGF receptor transactivation. Exp Cell Res 2005; 308: 241-253.

31. Puthalakath H, Villunger A, O'Reilly LA, Beaumont JG, Coultas L, Cheney RE et al. Bmf: a proapoptotic $\mathrm{BH} 3-$ only protein regulated by interaction with the myosin $\mathrm{V}$ actin motor complex, activated by anoikis. Science 2001; 293: 1829-1832

32. Suzuki $Y$, Rahman M, Mitsuya $H$. Diverse transcriptional response of CD4(+) T cells to stromal cell-derived factor (SDF)-1: cell survival promotion and priming effects of SDF-1 on CD4(+) T cells. J Immunol 2001; 167: 3064-3073.

33. Kook SH, Son YO, Jang YS, Lee KY, Lee SA, Kim BS et al. Inhibition of c-Jun N-terminal kinase sensitizes tumor cells to flavonoid-induced apoptosis through down-regulation of JunD. Toxicol appl pharmacol 2008; 227: 468-476.

34. Kuntzen C, Sonuc N, De Toni EN, Opelz C, Mucha SR, Gerbes AL et al. Inhibition of c-Jun$\mathrm{N}$-terminal-kinase sensitizes tumor cells to CD95-induced apoptosis and induces G2/M cell cycle arrest. Cancer Res 2005; 65: 6780-6788.

35. Ramjaun AR, Tomlinson S, Eddaoudi A, Downward J. Upregulation of two BH3-only proteins, Bmf and Bim, during TGF beta-induced apoptosis. Oncogene 2007; 26: $970-981$.

36. Prickett TD, Brautigan DL. Cytokine activation of $p 38$ mitogen-activated protein kinase and apoptosis is opposed by alpha-4 targeting of protein phosphatase $2 \mathrm{~A}$ for site-specific dephosphorylation of MEK3. Mol Cell Biol 2007; 27: 4217-4227.

37. Danial NN, Korsmeyer SJ. Cell death: critical control points. Cell 2004; 116: 205-219.

38. Labi V, Erlacher M, Kiessling S, Manzl C, Frenzel A, O'Reilly L et al. Loss of the BH3-only protein Bmf impairs $B$ cell homeostasis and accelerates gamma irradiation-induced thymic lymphoma development. J Exp Med 2008; 205: 641-655.

39. Okada N, Gao JQ, Sasaki A, Niwa M, Okada Y, Nakayama T et al. Anti-tumor activity of chemokine is affected by both kinds of tumors and the activation state of the host's immune system: implications for chemokine-based cancer immunotherapy. Biochem Biophys Res Commun 2004; 317: 68-76.

40. Akekawatchai C, Holland JD, Kochetkova M, Wallace JC, McColl SR. Transactivation of CXCR4 by the insulin-like growth factor-1 receptor (IGF-1R) in human MDA-MB-231 breast cancer epithelial cells. J Biol Chem 2005; 280: 39701-39708. 